\title{
Analysis of the security during power system expansion planning
}

\author{
Alexey Osak ${ }^{1, *}$, Daniil Panasetsky ${ }^{1}$, and Elena Buzina ${ }^{1}$ \\ ${ }^{1}$ Melentiev Energy Systems Institute, 130 Lermontov str., Irkutsk, Russia
}

\begin{abstract}
Increasing the intelligent level of the EPS control systems, caused by the implementation of Smart technologies, changes the structure and the properties of EPS and increases the importance of system reliability analysis. System reliability analysis includes two components - for the balance and for the regime. On the one hand, there is a large number of studies to assess the reliability of the power system, which examines various aspects and methods of solving this problem. On the other hand, in Russia there is no generally accepted methodology with clear criteria that could be used for feasibility studies of various technical solutions taking into consideration system reliability aspects. In practice, the security analysis is limited by the calculations of power flows, static and dynamic stability for a number of forecast periods for the normal and repair circuits considering the most severe disturbances. The existing approach allows defining the requirements and adjusting emergency control systems, but does not allow evaluating and comparing solutions for power grid constructions. The authors propose a new method for power system reliability evaluation, which is suitable for planning development and operation of power systems. The method includes a general description of the algorithm which allows to compare various development scenarios, as well as to assess the reliability level of their implementation. In particular, the method allows to determine where it is needed only the relay protection and emergency control system development, and where it is necessary grid, protection and control development and reconstruction.
\end{abstract}

\section{Introduction}

The rise of intelligent level of the power system (PS) control due to the implementation of Smart technologies and conception of intelligent power system (IPS) of active-adaptive grid (AAG) changes the structure and characteristics of PS [1-2], simultaneously increasing the importance of assessment of system reliability. System reliability (PS reliability) includes balance and regime components.

In Russia, in accordance with [3], power system security is understood as a property of the system to maintain the prescribed modes of operation when conditions change, element failures and sudden perturbations occur. In other countries, the term security has a similar meaning, which is understood as the ability of the system to withstand sudden disturbances [4-6]. There is distinguish between static and dynamic power system regime security. In assessing static regime security, dynamic transient processes in EPS are not taken into account, the model of the system is limited to the equations of steady-state. Dynamic regime security is related to the dynamics of EPS behavior, when a nonlinear model of transient processes in EPS or its linearized version can be considered.

There is a great number of studies on assessment of power system reliability (in particular [7-8]) dealing with various aspects and methods for solution of this problem.
On the other hand, in Russia there is no generally accepted methodology with clear criteria which could be used for development of inter- and intra-system connections while choosing the schemes of power generation at power plants and schemes of power supply of customers taking into account system reliability.

In practice, the security analysis is limited by calculations of static and dynamic stability for a number of forecast periods for normal and repair circuits considering the most severe disturbances [9]. Such an approach allows us to define requirements and adjust emergency control (EC). However, it cannot assess and compare solutions for power grid constructions.

The authors propose a new method to determine application of various aspects of security at different stages of development planning and during operation power system. A technique and algorithm are proposed that allow to determine the magnitude of possible undersupply of electricity for various emergency situations, taking into account the emergency control and the expected actions of the dispatcher. This approach allows us to determine the mathematical expectation of annual damage from all accidents in EPS, resulting in a shortage of electricity. 


\section{Methodology}

\subsection{Reliability criteria for power system expansion planning}

The most important criterion in planning of power system development is system reliability and PS viability. Moreover, both balance and regime aspects of system reliability should be provided [5-8]. Planning is carried out at various levels with different time interval. Therefore, various aspects of system reliability are considered for different types of planning (see Table 1). Measures for power grid construction are supported by an algorithm given in Fig. 1.

With regard to balance reliability, it is necessary to control it not only at the levels of power system (PS), unified power system (UPS) and integrated power system (IPS), but also at the level of large and medium power areas within PS and intersystem regions. At the level of UPS and PS, balance reliability is supported first of all by its own generation, whereas balance reliability of large and medium power areas is provided by transmission capacity of power grid connecting the power region with one or several PS. Total available reserve capacity (spinning and cold reserve capacity) of PS and adjoining PS and UPS is considered in assessment of balance reliability of PS or a power region. We distinguish two components in assessment of PS security:

Operational assessment of PS security during short-term post-emergency state of power system: evaluation of disturbance consequences (including static and dynamic stability analysis), operation of relay protection (RP), emergency control (EC), automatic voltage regulators (AVR), automatic frequency and power control (AFPC), sources of reactive power and on-load tap-changers (OLTC). Changes of generator power are recorded with primary regulation within the primary reserve.

Security during long-term post-emergency state of power system: evaluation of disturbance sequences implementing regime measures (20 and 40 minutes). Changes of generator power are recorded with primary, secondary and operational regulation within primary and secondary reserves.
Table 1: System reliability during different types of planning

\begin{tabular}{|c|c|}
\hline Reliability aspects & \begin{tabular}{|r} 
Reliability effect \\
\end{tabular} \\
\hline \multicolumn{2}{|c|}{ Long-term planning (over 10 years) } \\
\hline Balance reliability & $\begin{array}{c}\text { Measures for development of large } \\
\text { generation and power grid } \\
\text { construction of intersystem } \\
\text { connections }\end{array}$ \\
\hline \multicolumn{2}{|c|}{ Medium-term planning (1-10 years) } \\
\hline Balance reliability & $\begin{array}{c}\text { Planning of development of } \\
\text { generation and main power systems }\end{array}$ \\
\hline $\begin{array}{l}\text { Security of long-term } \\
\text { post-emergency state }\end{array}$ & $\begin{array}{c}\text { Planning of power grid construction ( } \\
\text { transmission lines, static var } \\
\text { compensator) }\end{array}$ \\
\hline Operational security & $\begin{array}{l}\text { Measures for EC development and, in } \\
\text { some cases, for power grid } \\
\text { construction (power generation at } \\
\text { nuclear power plants (NPP), some } \\
\text { thermal power plants (TPP) and } \\
\text { power supply of essential customers) }\end{array}$ \\
\hline \multicolumn{2}{|c|}{ Short-term planning (up to 1 year) } \\
\hline Balance reliability & $\begin{array}{c}\text { Planning of power grid and } \\
\text { generation repair and energy supply } \\
\text { for generation }\end{array}$ \\
\hline $\begin{array}{l}\text { Security of long-term } \\
\text { post-emergency state }\end{array}$ & $\begin{array}{c}\text { Planning of power grid and } \\
\text { generation repair }\end{array}$ \\
\hline Operational security & $\begin{array}{c}\text { Adjustment of RP, EC and ACS } \\
\text { devices }\end{array}$ \\
\hline \multicolumn{2}{|c|}{ Operational planning } \\
\hline Balance reliability & $\begin{array}{c}\text { Choice of composition and load of } \\
\text { operating power units }\end{array}$ \\
\hline $\begin{array}{l}\text { Security of long-term } \\
\text { post-emergency state }\end{array}$ & $\begin{array}{l}\text { Permit (ban) of joint repair and } \\
\text { realization of regime measures }\end{array}$ \\
\hline Operational security & Realization of regime measures \\
\hline
\end{tabular}

As a matter of priority, we assess the effect of these measures on the balance reliability of PS or power region during planning of power system development.

During planning of measures for power grid construction we assess the effect of these measures on the security in terms of long-term post-emergency PS state. Therefore, if security is not achieved because of some disturbances, we consider load limitation, energy undersupply and loss of lack of power overloading with corresponding probabilities of this disturbance, and feasibility of these or those measures for PS development is evaluated.

Finally, it is necessary to assess the effect of these measures on PS operational security during planning of power system development, including the comparison of some technical solutions, and during planning of measures for improvement of reliability of power grid within the framework of its operation.

To provide PS operational security, the following priority measures are applied: solutions of RP (including additional sets of the main protection and single-phase auto-reclosing with $220 \mathrm{kV}$ ), EC (including circuitbreaker failure protection caused by EC operation) and regime automation (including control of reactive power sources in the grid with $110 \mathrm{kV}$ and lower). Installation of additional sources of reactive power in the grid (including those on bus with $110 \mathrm{kV}$ and lower) is another measure for providing PS operational regime. Only in case of extremity, measures on additional power 
grid construction of new transmission lines (TL) are implemented for uninterrupted reliable power supply of customers or for supply of static or dynamic stability of NPP or TPP operation (if tripping of TPP energy units from EC causes long-term load limitation of customers).

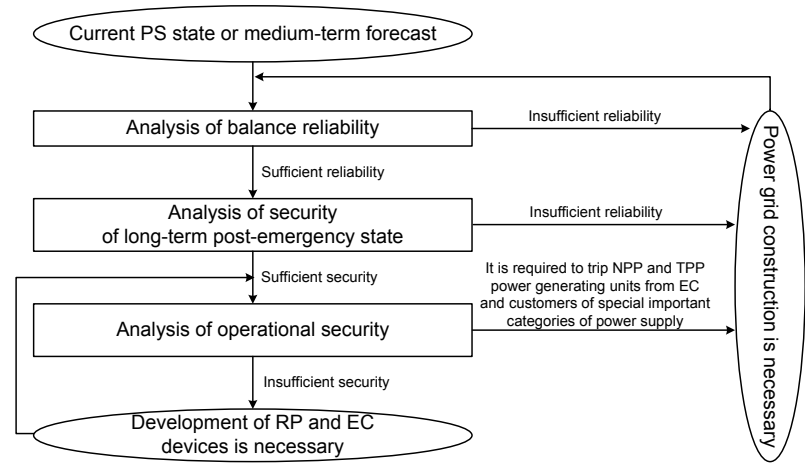

Fig. 1. Algorithm of feasibility studies for measures of power grid construction.

\subsection{Methods, assessment criteria and characteristics of PS security}

To assess PS security, we determine probability of reference disturbances [6] at different PS points. Reference disturbances should be subdivided into:

- Enumeration of reference disturbances with successful SPAR (single-phase auto-reclosing) / TPAR (three-phase auto-reclosing) or successful testing of voltage upon command of the operational-dispatcher personnel pointing to the probability for each elements of the grid (transmission line, transformer, power bus, power generating unit and 2 transmission lines). Data on reference disturbances are considered only in the analysis of PS security.

- Enumeration of reference disturbances with unsuccessful automatic and manual testing leading to long-term tripping of the element with probability for each element of the grid (grid element, power bus and power generating unit) as well as to the expected time of recovery. These reference disturbances are considered in the analysis of operational security and security during long-term post-emergency state of PS.

Different recovery (repair) time is needed for different emergency device damage. Therefore, besides medium duration of one repair and probability of emergency state of the grid element, it is recommended to predict probability of repairs of different duration. For example, repair time of one transmission line phase differs from time repair of transmission line pole with different probability of such damages. As for station equipment, time scale for reconstruction is larger, taking into account the fact that some damages need complete substitution of the damaged high-voltage equipment that could be either kept in the storehouse (emergency reserve when hours are needed for repair) or be absent (days or even weeks are needed for repair).

\subsubsection{Methods, assessment criteria and characteristics of security during long-term post- emergency state of $P S$}

We evaluate the ability of power system to resist disturbances associated with withdrawal of equipment for emergency repair taking into consideration the operation of RP, EC of automatic regulators and regime automatics and realization of regime measures by operational-dispatcher personnel during the first 20 (40) minutes after the first disturbance assessing the effect on power consumers.

At the moment of emergency disturbance by RP and EC operation, partial load shedding (power consumers) is possible as well as output of regime parameters (current, voltage and frequency) beyond permissible values. After RP and EC response, automatic regulators and regime automatics together with the operationaldispatcher personnel mobilize hot reserve at power plants. Regime parameters are inserted into permissible limits and power consumers disconnected during the emergency are connected. According to the rules [10, 11], it takes 20 minutes to carry out these measures. However, if this time is not enough, the forced regime of power system is introduced and other 20 minutes (totally 40 minutes from the beginning of the emergency) are spent for normalization of the post-emergency regime.

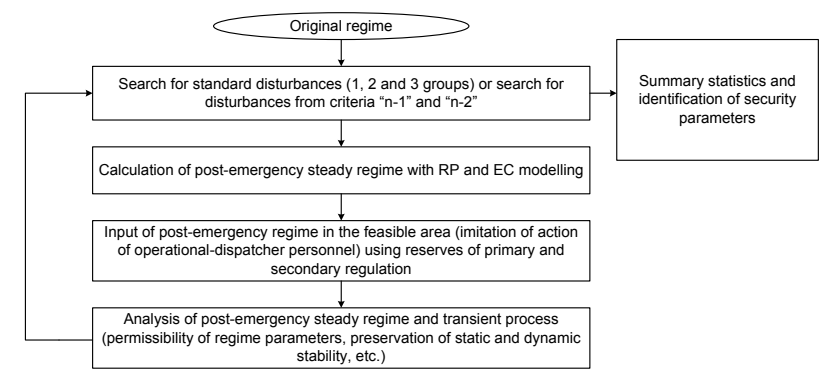

Fig. 2. Algorithm of security analysis during long-term postemergency state of PS

During this type of security the possibility of normalization of post-emergency regime is evaluated with switching on all consumers tripped for 20 (40) minutes during the emergency. If it is provided for concrete pre-emergency scheme-regime conditions and for concrete emergency disturbance, the security does not decrease. If limitations for consumers remain after 20 (40) minutes, we determine the volume and undersupply of electrical energy taking into account mobilization of all kinds of reserves.

Analyzing all disturbances (Fig. 2) and integrating probable volumes of electrical energy undersupply, the following algorithm is defined:

1. Original calculating model is developed. Preemergency values of loads and generations in the nodes and state of network elements are determined.

2. Available ranges are specified for regulation of variable regime parameters of static var compensator (SVC), on-load tap-changers (OLTC) of transformers and autotransformers (if automatic or operational tapchange operation is allowed), booster transformers (BT), 
fixed series compensation (FSC), thyristor-controlled series capacitor (TCSC), as well as generators with active and reactive power. These ranges can be as follows:

- For SVC:

- variable ranges of shunt of capacitor banks (Bmin, Bmax);

- variable ranges of reactive power (Qmin, $Q \max$ );

- type of regulation - smooth or discrete (a number of steps or values of changing parameter for each step of control);

- For longitudinal FSC (TCSC) and controlled longitudinal reactors:

- variable ranges of longitudinal resistance (Xmin, Xmax);

- type of regulation - smooth or discrete (a number of steps or values of changing parameter for each step of control);

- For OLTC of power and phase-variable transformers (autotransformers):

- variable ranges of transformation coefficient (Ktr.min, Ktr.max);

- a number or a step of changing of transformation coefficient;

- For generators:

- permissible values of reactive power ( $Q \min , Q \max$ or in the form of permissible power);

- permissible values of active power (Pmin, Pmax technological minimum and maximum);

- permissible values of changing of active power for 20- and 40-minute range (dPmin.20, dPmax.20, dPmin.40, dPmax.40).

3. Continuous permissible ranges are specified for regime limitations in nodes and branches of the calculating model:

- for voltage (Umin, Umax) and current (Imin, Imax).

4. Limitations are specified for capacity in the controlled sections:

- or in the form of values of maximum available load flow / emergency allowable load flow for concrete conditions of the grid elements (a list of switched on or tripped grid elements);

- or in the form of assignment of vector (trajectory) of weighting for calculation of maximum load flow (MLF) and emergency allowable load flow (EALF) of the concrete controlled section.

5. Controllers of EC device effect are specified:

- or with possible maximal volumes of decrease of load and generation;

- or with EC algorithms stating concrete controlling effects for each stage;

- load category is specified, which can be limited.

6. Possible controlling effects are specified for operational-dispatcher personnel (except specified in Item 2) in the following way:

- enumeration of possible maximal volumes of load shedding and generation of nodes with the help of - operational-dispatcher personnel;

- load category which can be limited.

7. Repair schemes are specified (for a concrete calculation interval: winter/summer etc.):

- or tripping of one grid element (under repair);
- or enumeration of changes of the grid topology (tripping of the equipment under repair, switching on/tripping of the section breakers, etc.), and switching on/tripping of the branches of the calculating model);

- or enumeration of the network topology changes and changes of generation composition (switched on/tripped - of generators and values of active generation power);

- in some cases, if the calculating model does not switch on distribution systems, besides changes of the grid topology and generation, the load redistribution between the nodes is specified (thus, changes of the distribution grid topology is considered including those of the grids of internal power supply of consumers with several supply centers).

8. Enumeration of emergency disturbances caused by long-term grid tripping is specified. Every disturbance is specified by:

- enumeration of tripped grid elements (branches of the calculating model, e.g. TL with tapping is modelled with several nodes and branches);

- probability of concrete emergency disturbance;

- expected time of recovery.

9. Search for emergency disturbances (Item 8) is specified for the original (normal) scheme and for all specified control schemes (Item 7) by turn:

- pre-emergency regime is assessed and changes are overlaid in the control schemes (Item 7, tripping/switching on of branches and changes of generation and load);

- emergency disturbances are searched by turn (Fig. 2) overlaying tripping specified for a concrete disturbance (Item 8);

- EC operation is modelled during estimation of the steady regime (Item 5, in case of its actuation for a concrete disturbance);

- if the iterative process is non-convergence during estimation of the steady regime, it is necessary to model EC (Item 5, using optimization method and method of directed or complete search) to reach post-emergency steady regime;

- if the obtained steady regime has load limitations (load shedding by EC, Item 5) and/or regime parameters go beyond permissible values (Item 3 ) or load flow along the controlled sections exceeds EALF (Item 4, specified or determined by the weighting for achieving postemergency regime), it is necessary to introduce the regime in the allowable area (Items 3 and 4), including the realization of measures available for operationaldispatched personnel (Items 2 and 6) and if possible to recover the load tripped from EC (Item 5);

- if post-emergency regime together with realization of input measures meets all limitations (Items 3 and 4) and if the load tripped from EC but during the input it was possible to recover it, such disturbance does not reduce security;

- if post-emergency regime together with realization of input measures does not meet all limitations (Items 3 and 4 ), it is necessary to fulfil additional adjustment of control able to be applied by operational-dispatcher personnel (Item 6);

- if post-emergency regime together with realization of input measures meets all limitations (Items 3 and 4) but 
has non-regenerable load (dPlim.), it is necessary to calculate electrical energy undersupply (dWlim). Duration of electrical energy undersupply is calculated from the expected time of recovery of the tripped emergency element (Trep.), which can be decreased by time of generation mobilization (Tmob), if all permissible values of changes of active power for 40minute range or together with mobilization of cold reserve (limitations on technological maxima and minima) allow completely or partially recovery of preemergency level of load.

10. Integral parameters of electrical power undersupply and damage together with probability are calculated from results of search for all disturbances for all calculating models. Moreover, category of consumers included in the limitation list is taken into consideration.

11. If several options of the development of power grid are compared, parameters specified in Item 10 are calculated for each option.

Item 9 points to the necessity of applying methods imitating EC operation as well as necessity of using methods of input into the allowable area [12-13]. Moreover, the result obtained should be balanced steady regime (the first and second Kirchoff's laws are followed), as well as limitations stated in Items 2, 5 and 6 and Items 3 and 4 for input into the allowable area are taken into consideration.

\subsubsection{Methods, assessment criteria and parameters of operational security}

Operational security is an aspect of system reliability reflecting ability of power system to resist sudden disturbances taking into account operation of RP, EC, ACS and automatic regulators without unforeseen effects on power consumers. Operational control includes static and dynamic security. Dynamic transitional processes in PS are not taken into account during assessment of static security. The system model is limited by equations of the steady regimes. Dynamic security is connected with dynamics of PS behavior. Moreover, nonlinear model of transitional processes or its non-linearized variant occurring in PS can be considered.

Priority measures for providing operative security of PS are solutions of RP (including those of additional sets of the main protection and SPAR with $220 \mathrm{kV}$ ), EC (including CBFP caused by EC), regimes automatic control device (including control of the available sources of reactive power with $110 \mathrm{kV}$ and lower). All necessary devices of RP, EC and ACS have to be installed in full volume during planning of development and operation of power systems. All these devices do not have to permit disturbances of static or dynamic stability of PS during emergence of standard disturbances and also nonpermissible in value and duration overload of devices, as well as deviations of voltage and frequency. Optimal options of realization of RP, EC and ACS should be chosen within the framework of fulfilment of the project work during construction and reconstruction of energy facilities.
In terms of long-term post-emergency state of PS, the analysis of security in accordance with the proposed algorithm and scheme of power generation at NPP and TPP allows us to reveal long-term limitation of power load for customers because of long period of putting into operation the power unit tripped from EC. In these cases, we need such a scope of power grid construction, which in combination with effective and sufficient RP and EC devices would not permit tripping of NPP or TPP power units cause by disturbance of static or dynamic stability during standard disturbances. Moreover, during standard disturbances EC (automatic stability control system or automatic overload protection) can use controlling effects that are not associated with tripping of power units, such as CBFP from EC, control of SVC from EC, impulse and long-term unload of turbines, etc.

If reliability of power supply of consumers with special needs for reliability and continuity of power supply is under consideration, possibility of their tripping from EC or under command of operationaldispatcher personnel is not foreseen. Uninterruptedness of power supply of such consumers is provided if necessary by additional power grid construction (including by SVC device), RP, EC and ACS devices.

\subsection{Summary criteria (standards) of evaluation and parameters of security}

To determine damage caused by electrical power undersupply, it is suggested to assess it from power capacity of gross domestic product (GDP) for the country as a whole and from power capacity of gross regional product (GRP) for concrete entities of the Russian Federation. GDP and GRP values as well as their quantitative parameters of the power consumed within the entire country and within the concrete region are quite objective criteria with high degree of their validity. Therefore, mean specific damage from the electrical power undersupply is determined from the equations:

$$
\begin{aligned}
& Y_{0 . R F}=G D P_{R F} / W_{R F \text { year }} \\
& Y_{0 . \text { Reg }}=G R P_{\text {Reg }} / W_{\text {Reg year }}
\end{aligned}
$$

It is possible to introduce a multiplying coefficient in the equations (1) and (2) that take into consideration additional time and resource costs of power supply consumers for resumption of their economic activity after power restoration (lifting of limitations). However, due to complexity of the grounds for concrete values increasing the coefficient, it is recommended not to introduce them. For large or particularly critical industrial value of the damage is determined individually.

The duration of electrical power undersupply ( $T_{\text {lim.emerg. }}$ No. $x x x$ ) for a concrete emergency situation (see Item 2.2.1.) is determined from the minimum of mean repair time of the damaged device $\left(T_{\text {rep. emerg. No. } x x x}\right)$ or mean time of mobilization of the generating power $\left(T_{\text {mob. emerg. }}\right.$ No. $x x x)$ :

$T_{\text {lim. emerg. No. xxxx }}=\min \left(T_{\text {rep. emerg. No. xxx }} T_{\text {mob. emerg. No. xxx }}\right)$ 
Therefore, the value of electrical power undersupply $\left(d W_{\text {lim. emerg. No. } x x x}\right)$ for a concrete emergency situation is determined from the value of power limitation $\left(d P_{l i m}\right.$. emerg. No. $x x x)$ and duration of power undersupply ( $T_{\text {lim. emerg. }}$ No. $x x x$ ):

$$
\left.d W_{\text {lim. emerg. No. } x x x}=d P_{\text {lim. emerg. No. } x x x} \cdot T_{\text {lim. emerg. No. } x x x}\right)
$$

The expectation value of the annual monetary loss (ML) from the concrete situation $\left(M L_{\text {ann. emerg. No. } x x x}\right)$ depends on failure flow for a year for concrete damaged equipment during this emergency ( $w_{\text {emerg. No. } x x x}$ ):

$$
\begin{aligned}
& M L_{\text {ann. emerg. No. } x x x}=w_{\text {emerg. No. } x x x} \cdot d W_{\text {lim. emerg. No. } x x x} Y_{0 . R F} \quad(5 a) \\
& M L_{\text {ann. emerg. No. } x x x}=w_{\text {emerg. No. } x x x} d W_{\text {lim. emerg. No. } x x} Y_{0 . \text { Reg. }}(5 \sigma)
\end{aligned}
$$

The expectation value of the annual loss from all emergencies in the power system associated with the electrical power undersupply will be determined from the arithmetical sum of expectation values of annual losses from concrete emergencies $\left(M L_{\text {ann. emerg. No. xxx }}\right)$ :

$$
M L_{\text {ann.emerg }}=\sum_{i=1}^{n} M L_{\text {ann.emerg №i }}
$$

Besides the loss of the expected electrical power undersupply, it is necessary to take into account a probable loss from unforeseen tripping of consumers or generation. It is suggested to consider this loss only in those cases and volumes which are stipulated in the agreements (agreements of power supply or technological connection). If these aspects are not stipulated in the agreement, the loss is not considered. The maximum allowed time of electrical power undersupply for concrete consumers should correspond to agreement conditions and category of consumers (according to the decrees of the Russian Federation Government).

\section{Conclusion}

This paper presents methods for assessment of system reliability during planning of power system development. The method proposed contains general description of the step-by-step algorithm with the help of which it is possible to compare options and scenarios of power system development and to assess the level of reliability control for validation of decision making.

The most significant assessment of security is the evaluation of losses during long power limitation of consumers (over 20 and 40 minutes). The algorithm proposed allows the calculation of probable losses for comparative analysis of different options of PS development. Operational security should be provided, first of all, with adequate and effective RP and EC devices. Only in those cases when even short-term tripping of generation or consumers by EC operation are unacceptable (due to specific conditions), security should be provided with the power grid construction.

\section{References}

1. N.I. Voropai, D.N. Efimov et al., Smart Grid, IEEE Transactions on. Vol. 4, no. 3. P. 1732-1740. (2013)

2. N.I. Voropai, A.B. Osak, Energy Policy. No. 5. P. 60-63. (2014).

3. Eds. N.I. Voropai, Reliability of energy systems (Collection of recommended terms) (Moscow: "Energia", 2007. 192 p).

4. Reliability concepts in bulk electric power systems (New York: North American Electric Reliability Council, 1985. 96 p).

5. N. Balu, T. Bertram, A, Bose et al., Proceedings of the IEEE. Vol. 80, No. 2. P. 262-280. (1992).

6. R.J. Marceau, J. Endrenyi, R. Allan et al., Electra. No. 175. P. 49-77. (1997).

7. Ed. N.A. Manov, Methods and models for studying power system reliability (Syktyvkar: Komi SC UrB RAS. 2010. 292 p).

8. Eds. N.I. Voropai, G.F. Kovalev, Concept of reliability control in electric power engineering (M.: LLC PH "Energy". 2013. 304 p).

9. Guidelines for stability of power systems, approved by Order No. 277 of 30.06.2016 of Russian Ministry of Energy.

10. STO 59012820.29.240.007-2008. Rules for prevention of development and disturbance liquidation of the normal state of electrical annex of power systems. Standard JSC "SO UPS".

11. STO 59012820.27.010.002-2016. Rules of transition to operation in forced regime in controlled sections of the dispatcher center JSC SO UPS, Standard JSC "SO UPS".

12. A.B. Osak, A.I. Shalaginov, D.A. Panasetsky, E.Ya. Buzina, Book of reports of international conference "Modern tendencies of System Development of Relay Protection and Automation of Power System", Sochi. 15 June 2015.

13. A.B. Osak, A.I. Shalaginov, D.A. Panasetsky, E.Ya. Buzina, Methodic problems of studies of reliability of large power systems, Syktyvkar: LLC "Komi Republican Printing House”, p. 337-346. (2016). 